\title{
THE CHEMICAL EVOLUTION OF LIGHT ELEMENTS IN OUR GALAXY
}

and some implications for "Cosmic Chemical Evolution"

\author{
N. PRANTZOS
}

Institut d'Astrophysique de Paris

98bis, Bd. Arago, 75014 Paris

\section{Introduction}

Progress in the theory of galactic chemical evolution has been very slow and it is only in the solar neighborhood that observations constrain seriously the parameters of the various models. The history revealed on the basis of these data allows only for a small depletion of deuterium (D), less than a factor of 3 from its pregalactic value (Sec. 2.1). The observational data for the rest of the Milky Way disk are much less constraining for the models. They suggest, however, that a much larger astration (and, hence, D depletion) has taken place in the inner Galaxy; the resulting D gradient, measurable by the future FUSE-LYMAN mission, should provide invaluable information as to the past history of the disk (Sec. 2.2). Also, assuming that our Galaxy is a typical spiral, one can calculate the properties of disk galaxies as a function of redshift (in the framework of a given cosmological model) and compare to the observed properties of the extragalactic universe: global star formation rate, gas content and metal abundances in gas clouds. It turns out that D can be considerably depleted in galaxy disks, but only at low redshifts (Sec. $2.3)$.

Observations of the light element $\mathrm{Be}$ and $\mathrm{B}$ abundances in halo stars show a surprising linear dependence on metallicity. Despite recent theoretical attempts, this linearity still defies a simple theoretical explanation (Sect. 3), but certainly has important implications for our understanding of cosmic ray acceleration and composition. 

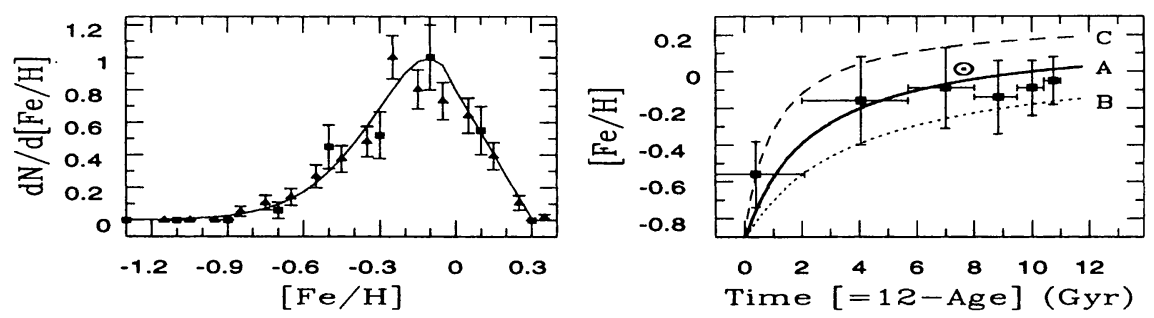

Figure 1. Observables in the solar neighborhood.

a: Metallicity distribution of G-type stars, from 2 recent surveys [25, 34]. The solid line is a fit to the data.

b: Age-metallicity relationship of F-stars, from [5]. The data (182 stars) are binned in groups of 0.2 in $\log (\mathrm{Age} / \mathrm{Gyr})$; instead of Age, Time=12-Age is plotted here. Metallicities are for stars with galactocentric distances evaluated to $8-9 \mathrm{kpc}$ (i.e. appropriate to local disk) and volume corrected, where the vertical error bars represent $1 \sigma$ dispersion in metallicity for each age group (Col. 6 of Tables 14 and 15, respectively, in [5]). The 3 cases $\mathrm{A}, \mathrm{B}$ and $\mathrm{C}$ trace schematically the mean metallicity and the observed dispersion. Notice that simple, one-zone, chemical evolution models always yield a unique age-metallicity relationship and cannot reproduce that scatter without furher assumptions and free parameters.

\section{Deuterium evolution in the Milky Way and in high redshifts}

\subsection{THE PAST HISTORY OF THE SOLAR NEIGHBORHOOD}

In the local disk (LD, defined as a cylinder of $\sim 1 \mathrm{kpc}$ radius at a distance $R_{\odot}=8.5 \mathrm{kpc}$ from the galactic center), the main observables relevant to chemical evolution are $([20,16])$ :

- the current surface densities of gas, stars and total amount of matter ( $\sim 10,40$, and $55 \mathrm{M}_{\odot} \mathrm{pc}^{-2}$, respectively), leading to a current gas fraction of $\sigma_{G} \sim 20 \%$; also, the current star formation rate (SFR), $\mathrm{S}_{0} \sim 1-3$ $\mathrm{M}_{\odot} \mathrm{pc}^{-2} \mathrm{Gyr}^{-1}$.

- the abundances of various elements in the ISM today $\left(\mathrm{Z}_{0}\right)$ and 4.5 Gyr ago, the latter presumably represented by the solar system ones $\left(\mathrm{Z}_{\odot}\right)$.

- the oxygen vs. $\mathrm{Fe}(\mathrm{O}-\mathrm{Fe})$ relationship, showing a steady decline [from $\mathrm{O} / \mathrm{Fe} \sim$ const. $\sim 3$ times solar at $[\mathrm{Fe} / \mathrm{H}]<-1$ (i.e. during the halo phase), to $\mathrm{O} / \mathrm{Fe} \sim$ solar at $[\mathrm{Fe} / \mathrm{H}] \sim 0]$, usually attributed to the delayed ( $\sim 1 \mathrm{Gyr}$ ) appearance of SNIa, producing $2 / 3$ of the galactic Fe.

- the age-metallicity (Z-t) relationship, traced by the Fe abundance of long-lived, F-type stars.

- the metallicity distribution of long-lived G-type stars (Fig. 1a), showing that very few of them were formed at $[\mathrm{Fe} / \mathrm{H}]<-0.7(1 / 5$ solar $)$.

The last two observables allow to recover the past SFR history of the 

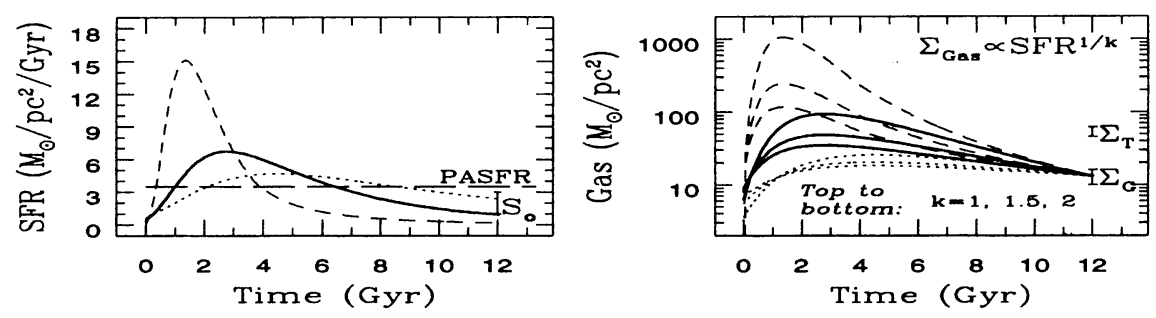

Figure 2. Observationally derived Star Formation Rate in the solar neighborhood. a: The 3 curves correspond to cases $\mathrm{A}, \mathrm{B}$ and $\mathrm{C}$ of Fig. $1 \mathrm{~b}$; they are all normalised to $\int_{0}^{T} S F R(t) d t=42 \mathrm{M}_{\odot} \mathrm{pc}^{-2}$, with $\mathrm{T}=12 \mathrm{Gyr}$. Case $\mathrm{C}$ leads to unacceptably low current SFR $\sim 0.1 \mathrm{M}_{\odot} \mathrm{pc}^{-2} \mathrm{Gyr}^{-1}$, much lower than the observed $\mathrm{S}_{0} \sim 1-3 \mathrm{M}_{\odot} \mathrm{pc}^{-2} \mathrm{Gyr}^{-1}$.

b: Past history of the gas surface density, assuming SFR $\propto \Sigma_{G}^{k}$. Te 3 types of curves (solid, dotted, dashed) correspond again to those of Fig. 1b and 2a. Case C violates the constraint $\Sigma_{G}<\Sigma_{T}$ for all plausible values of $k$.

local disk $([23,22])$, as:

$$
S F R \propto \frac{d N_{G}}{d t}=\frac{d N_{G}}{d[F e / H]} \frac{d[F e / H]}{d t}
$$

where the assumption of a constant IMF is necessary for the proportionality between the SFR and the creation rate of G-stars to hold. The scatter in the Z-t relationship does not allow to recover unambiguously the SFR. For illustration purposes, we adopt the 3 curves of Fig. 1b, which have considerably different early and late slopes and cover most of the observed dispersion. When the resulting SFR histories are normalised to the current local star surface density $\Sigma_{*}$ over the age $\mathrm{T}=12 \mathrm{Gyr}$ of the galactic disk $\left(\int_{0}^{T} S F R(t) d t=\Sigma_{*}\right)$, only cases A and B lead to an acceptable current SFR; case $\mathrm{C}$, as well as all other Z-t relationships with large early slopes, lead to a large early star formation and to too low current SFR (Fig. 2a).

There is another indication against C-type cases. Observations suggest that in disk galaxies $\mathrm{SFR} \propto \Sigma_{G}^{k}$, with $k=1-2$ (e.g. [9]). Knowing the local SFR history from eq. (1), one can then recover the gas history as $\Sigma_{G} \propto \mathrm{SFR}^{1 / k}$, normalising to the current $\Sigma_{G}$. It can be seen from Fig. $2 \mathrm{~b}$ that $\mathrm{C}$-type cases should be excluded, since they lead to past $\Sigma_{G}$ larger than the current total surface density $\Sigma_{T}$.

The above results indicate that the local disk was formed on timescales of many Gyr. Models with slow infall of primordial composition reproduce reasonably well the above constraints. Such models ([21, 31] and Fig. 3) deplete $\mathrm{D}$ only by factors $\sim 2-3$, at least for reasonable stellar Initial Mass Functions (IMF), like the one of [10]. Notice, however, that the main reason for the small D depletion is the currently large local gas fraction $(\sim 20$ $\%$ ), showing that a rather large fraction of the ISM has not been astrated 

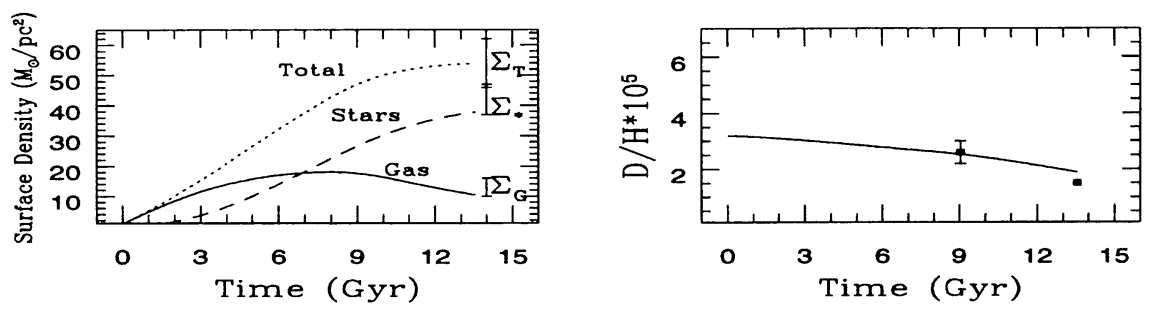

Figure 3. Results of a model for the chemical evolution of the local disk.

a: Evolution of the gaseous, stellar and total mass and comparison of the final results to observations of current local $\Sigma_{G}, \Sigma_{*}$ and $\Sigma_{T}$, respectively. The adopted SFR is proportional to $\Sigma_{G}^{1.5}$, while the infall rate is a broad gaussian with $\Delta \tau=6 \mathrm{Gyr}$, i.e. the local disk is formed very slowly. Such models reproduce reasonably well the observational constraints of Sec. 2.1 and Figs. 1 and 2a.

b: Corresponding evolution of the $\mathrm{D}$ abundance, compared to observations at solar system formation ([8]) and in the local ISM (Linsky, this volume). Only small factors of $\mathrm{D}$ depletion $(2-3)$ are obtained in this kind of models, with slow infall of primordial composition.

yet. These results point to low values for pregalactic $\mathrm{D} / \mathrm{H} \sim 3-410^{-5}$, i.e. compatible with those advocated by Tytler (this volume). Those values also alleviate (but do not solve) the problem of ${ }^{3} \mathrm{He}$ overproduction, which is definitively a stellar nucleosynthesis, not a galactic evolution, problem (see [7] for possible solutions).

\subsection{THE EVOLUTION OF THE MILKY WAY DISK}

Contrary to the case of the solar neighborhood, the available observations for the Milky Way disk offer information mainly about its current status, not its past history. The main relevant observables are ([20]):

i) The total mass of gas and stars in the disk $\left(\sim 810^{9} \mathrm{M}_{\odot}\right.$ and $\sim 510^{10}$ $\mathrm{M}_{\odot}$, respectively); the total current SFR $\left(\sim 3-5 \mathrm{M}_{\odot} \mathrm{yr}^{-1}\right)$ and the current supernova rates $(\sim 2 \mathrm{SNII} /$ century and $\sim 0.4 \mathrm{SNIa} /$ century, respectively, as suggested by observations of external spirals).

ii) The current gas and stellar profiles, showing that the gas fraction profile steeply decreases in the inner disk and suggesting that the SFR efficiency has been larger in those regions than in the outer disk.

iii) The current SFR profile, strongly decreasing outwards, but never proportional to some power of the gaseous profile alone.

iv) The current metallicity profile, traced by oxygen observed in HII regions, planetary nebulae and B-type stars and showing a gradient of $\mathrm{d}[\mathrm{O} / \mathrm{H}] \sim-0.07 \mathrm{dex} / \mathrm{kpc}([27,28])$.

These constraints make the Milky Way disk a "testbed" for theories of star formation in disk galaxies. The results of a simple model appear on 

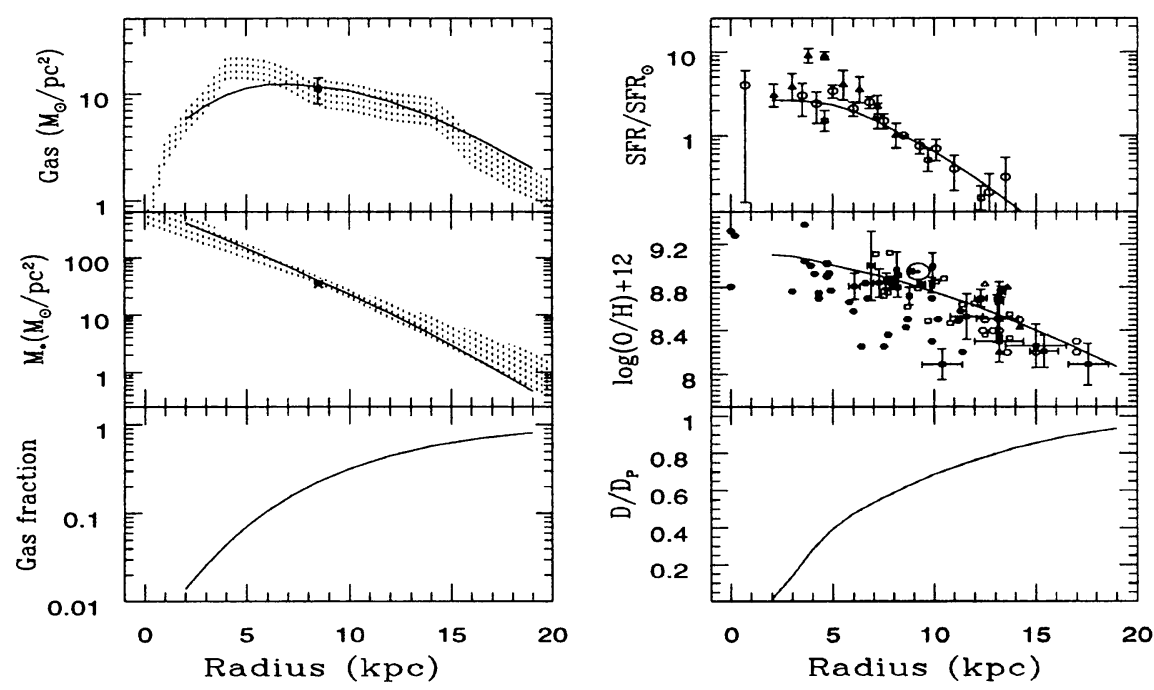

Figure 4. Results of a simple (independent ring) model for the chemical evolution of the Milky Way disk at a galactic age $\mathrm{T}=13 \mathrm{Gyr}$, and comparison to observations. The adopted SFR is SFR $\propto \Sigma_{G}^{1.5} / \mathrm{R}$ and the adopted infall rate is gaussian in time with $\Delta \tau=6$ Gyr in all the zones.

Left, from top to bottom: final profiles of the surface density of gas, stars and of the gas fraction, respectively. Solid lines: model results; shaded regions correspond to observations for the disk, and data points at $R=8.5 \mathrm{kpc}$ to solar system values.

Right, from top to bottom: current SFR, O and D profiles, respectively. SFR is normalised to its local value and $\mathrm{D}$ to its primordial one $\mathrm{D}_{P}$. Data for $\mathrm{O}$ are from $\mathrm{HII}$ regions (open symbols, from $[27,6]$ ) and B-stars (filled symbols, from [28]).

Fig. 4. The adopted SFR $\propto \Sigma_{G}^{1.5} / R$ is based on the idea that star formation in disk galaxies is induced by the propagation of spiral waves ([33]). The radial variation in the SFR efficiency is the only new parameter introduced in the model, all the others been already fixed by the modelisation of the solar neighborhood (Sect. 2.1). It turns out that with this simple parametrisation the model reproduces reasonably well the constraints (i-iv); it can be used then with some confidence for making further predictions. The most important result of this type of models (in the context of this Conference) concerns the evolution of deuterium. It is found that its final abundance profile shows an important gradient, much more important than (and anticorrelated to) the one of oxygen. The $\mathrm{D}$ abundance profile turns indeed to be the most sensitive tracer of the past SFR history of the disk ([21]), especially in the inner regions. Measurements of the D profile with the ISO satellite and the forthcoming FUSE-LYMAN mission should give invaluable information about the past star formation activity in the disk. 


\subsection{IMPLICATIONS FOR "COSMIC CHEMICAL EVOLUTION"}

In recent years, observations of high red-shift systems started revealing some views of the early phases of chemical evolution in the Universe. These observations concern: i) the "global" SFR ([11]); ii) the HI content of the Universe ([29]) and iii) the abundances of various elements in gaseous systems, in the line of sight of quasars $([15,18])$. To these one should include observations of $\mathrm{D}$ abundances, although the current status of those observations does not allow to establish a trend as a function of redshift.

Observables (a-c) have prompted several studies of "cosmic chemical evolution" (e.g. [17]). Notice however that, although observables (a) and (b) can be considered as truly "global", observable (c) is rather "local": indeed, it traces the metallicity of only those systems that have still retained some gas at a given redshift. The metals produced e.g. early on in ellipticals and already incorporated in their stars or expelled as ionised gas in the intergalactic medium of galaxy clusters, do not appear in the "census" of (c). The predictions of the "global" model for the evolution of the metallicity cannot then be directly compared to the observations in (c).

Since the only system with data sufficient to constrain its history is the Milky Way, it may be interesting to see how its evolution, modelled in Sect. 2.2 and translated in a cosmological framework, compares to the observables (a-c) of "cosmic evolution". The aim is to see to what extent the Milky Way evolution differs from the "average" one ([22]).

The results are shown in Fig. 5. Milky Way type spirals can account only partially for the observed evolution of the cosmic SFR; this is a reasonable conclusion, since other galaxy types (ellipticals? mergers? earlier type spirals?) are also expected to have a large contribution to the cosmic SFR at $z>1$. The interesting result of that exercice is that the evolution of $\mathrm{Zn}$ in the various disk zones brackets well the observations as a function of the redshift. It may well be indeed, that most of the lines of sight to quasars intercept various parts of (proto)galactic disks. This conclusion is opposite to the one reached in [15], which was based on a comparison of the data to the age-metallicity relationship of the LD only. Notice that for geometrical reasons, the probability of detecting outer disk regions is higher, favouring systematically lower abundances than those spanned during the LD history.

Finally, in the framework of the same model, it turns out that large D depletion may indeed take place in inner galactic disks, but only at low redshifts. $\mathrm{D}$ abundances at high redshifts in disk galaxies should always be close to the primordial value. If the observed values differ indeed by the large factors reported in this meeting (Tytler and Lanzetta, respectively), then at least one of the corresponding systems has evolved in a radically different way from a typical spiral. 

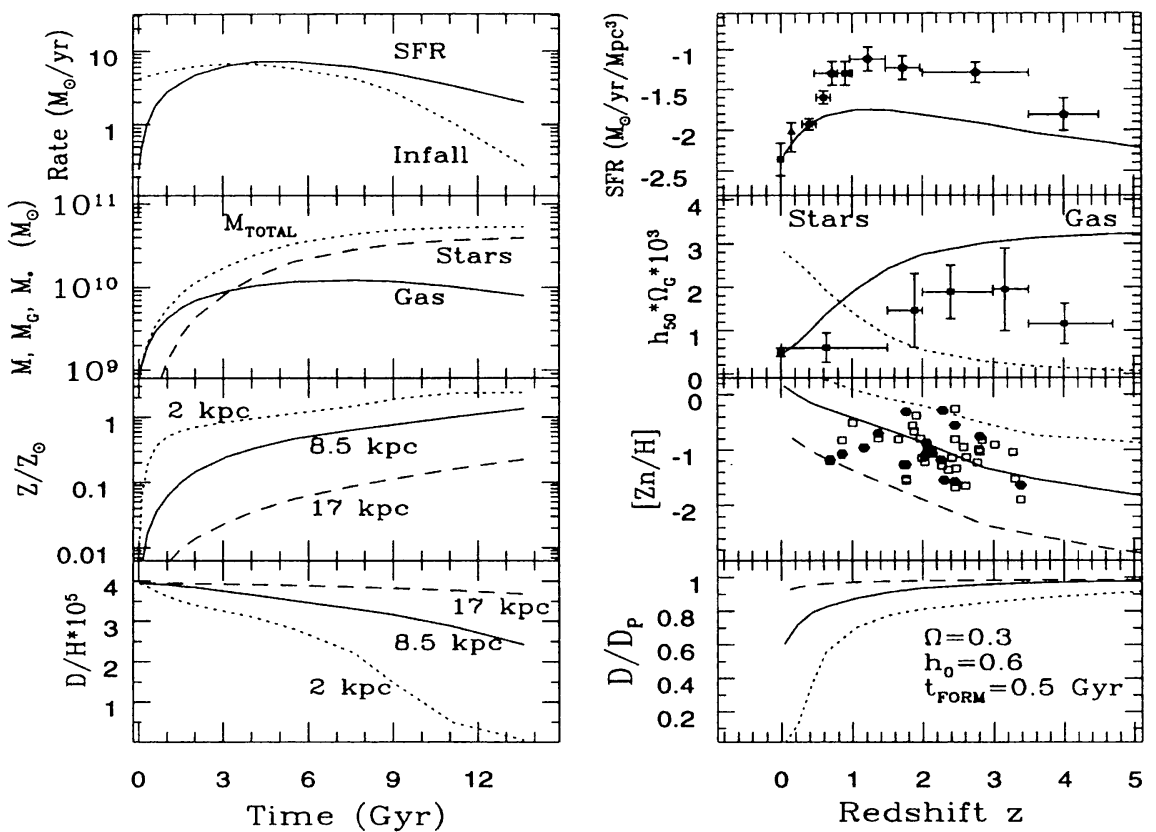

Figure 5. History of the Milky Way disk as a function of time (left) and of redshift (right), assuming that our galaxy is a typical spiral; a cosmological model with $\Omega=0.3$, $\mathrm{h}_{0}=0.6$ and galaxy formation starting $0.5 \mathrm{Gyr}$ after the Big Bang is adopted.

Left, from top to bottom: History of a) SFR and infall rate ; b) gaseous, stellar and total mass ; c) overall metallicity in three different zones, at distances of $2,8.5$ and $17 \mathrm{kpc}$ from the galactic center; d) D abundance in the same regions.

Right, from top to bottom: a) the "cosmic" SFR of disk galaxies, when normalised to the current local value $(z=0)$, does not show the steep observed increase back to $z \sim 1$ (although it peaks at $z \sim 1$, as observational data do); other galaxy types (ellipticals ?) should account for the discrepancy between theory (solid line) and observations (data from [11]) b) Evolution of gas and stars; data for HI gas density from [29]. c) The evolution of $\mathrm{Zn} / \mathrm{H}$ in various regions of spiral disks (only three are shown here, corresponding to those on the left) brackets well the observed abundances of $\mathrm{Zn} / \mathrm{H}$ in Ly $\alpha$ absorbers (data from $[18,15]$ ); those systems may well be (proto)galactic disks. d) The corresponding evolution of $\mathrm{D}$ shows that considerable depletion may take place in the inner disk regions, but only at low redshifts; abundances measured at high redshifts should be close to the primordial value.

\section{Evolution of Be and B in the Galaxy}

Thermonuclear reactions in stars cannot synthesize the light isotopes ${ }^{9} \mathrm{Be}$, ${ }^{10} \mathrm{~B},{ }^{11} \mathrm{~B}$, and ${ }^{6} \mathrm{Li}$. These, along with part of ${ }^{7} \mathrm{Li}$, are thought to be produced by spallation reactions of $\mathrm{CNO}$ nuclei during the propagation of cosmic rays (CR) in the Galaxy (e.g [24]). Indeed,the observed CR flux, integrated over $10^{10}$ years of galactic evolution, seems sufficient to produce the solar abun- 
dances of $\mathrm{B}, \mathrm{Be}$ and ${ }^{6} \mathrm{Li}$ (but only $\sim 10 \%$ of the solar ${ }^{7} \mathrm{Li}$, for which some other source, like AGB stars or novae, is required). Adopting CR spectra similar to those observed in near-Earth space (i.e. for energies $E>100$ $\mathrm{MeV} /$ nucleon, since lower $\mathrm{E}$ particle fluxes are supressed by the solar wind), the models are able to reproduce the solar system $\mathrm{B} / \mathrm{Be}$ and ${ }^{6} \mathrm{Li} / \mathrm{Be}$ ratios, but produce low ${ }^{11} \mathrm{~B} /{ }^{10} \mathrm{~B} \sim 2.5$ ratios compared to the observed one $(\sim 4)$. Large fluxes of $<100 \mathrm{MeV} /$ nucleon $\mathrm{CR}$ particles have been postulated to solve that discrepancy ([13]), the behaviour of the corresponding spallation cross-sections at low energies favouring the production of ${ }^{11} \mathrm{~B}$. More recently, $\nu$-induced nucleosynthesis in SNII has also been suggested as a supplementary source of ${ }^{11} \mathrm{~B}$ ([32]).

The evolution of $\mathrm{Be}$ and $\mathrm{B}$ received considerable attention in the 90ies, since observations of their abundances in halo stars $([26,3])$ showed that: a) contrary to $\mathrm{Li}$, they show no "plateau" value at low metallicity, i.e. no indication for a primordial origin; $b$ ) they evolve with a constant ratio of $\mathrm{B} / \mathrm{Be}$ 10-15, a clear indication of $\mathrm{CR}$ origin; c) their relationship w.r.t. Fe is a linear one, over three decades of metallicity. This latter observable is difficult to interpret in the framework of current ideas about CR origin. Indeed, the dominant current component of $\mathrm{BeB}$ synthesis $\left(\mathrm{p}, \alpha_{C R}+\mathrm{CNO}_{I S M}\right.$, accounting for $70-80 \%$ of the total) is of secondary origin, since the abundance of parent $\mathrm{CNO}_{I S M}$ nuclei increases with metallicity; despite some theoretical efforts $([26,19])$, this component cannot account for the observed linearity.

Attention was then focused on the currently minor mode of BeB synthesis $\left(\mathrm{CNO}_{C R}+\mathrm{p}, \alpha_{I S M}\right)$. If the abundances of $\mathrm{CNO}_{C R}$ remain $\sim$ const. in time, this mode may produce primary $\mathrm{BeB}([3])$ and dominate for $\mathrm{Z}<\mathrm{Z}_{\odot} / 5$. High energy (HE) CR show a source composition similar to the ISM one, i.e. their metallic content is expected, in principle, to evolve in time. However, their observed excess of $\mathrm{C}$ and ${ }^{22} \mathrm{Ne}$ is attributed to the contribution of material from the He-burning zones of massive stars, expelled through the Wolf-Rayet (WR) stellar winds ([14]); this material has no time to mix with the ISM before the subsequent SN explosion and may constitute a source of primary BeB. Similar conclusions hold if there exist large scale fluxes of low energy (LE) CR particles in the Galaxy rich in $\mathrm{C}$ and $\mathrm{O}$ ([2]), a rather strong generalisation of the inferrence made from the recent observations ([1]) of localised 3-7 MeV $\gamma$-ray emission from Orion.

Could WR stars provide the source of "metallic" CR for primary Be and $B$ ? Unfortunately, the WR phenomenon itself depends heavily on metallicity, as indicated by statistics of WR/O and WC/WR numbers (WC being the sub-type with the He-burning products at the surface) in regions of different metallicities; this is attributed to the reduced effect of radiation pressure on metal-poor stellar envelopes (the main WR formation mecan- 

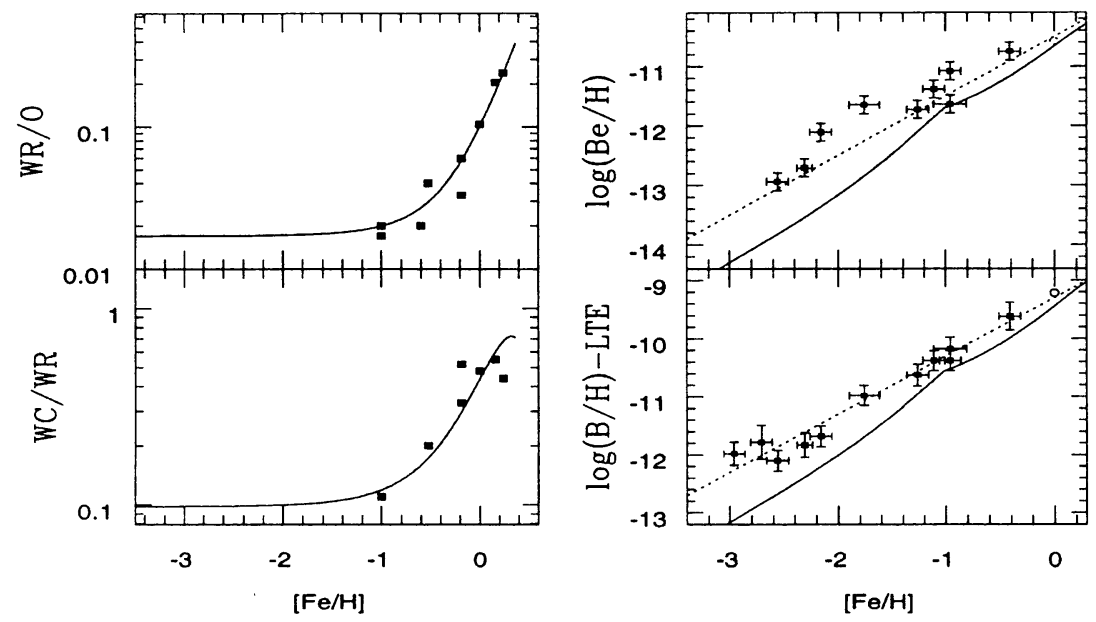

Figure 6. Statistics of WR and WC stars (left) and effect of a primary $\mathrm{CNO}_{C R}$ component resulting from WC stars on the evolution of Be and B (right).

Left: The solid curve is a fit to the $[\mathrm{Fe} / \mathrm{H}]>-1$ data points (from [12]) and a most optimistic extrapolation at $[\mathrm{Fe} / \mathrm{H}]<-1$.

Right: Model results (solid curve) compared to data (from [4]) and to a slope $=1$ (dotted) curve.

ism for solar metallicity single massive stars). Roche-lobe overflow in binary stars offers an alternative, metallicity independent, mechanism for WR formation but, as seen in Fig. 6, it cannot create more than $\sim 10 \%$ of the solar $\mathrm{WC} / \mathrm{O}$ ratio.

Assuming that WR stars produce primary Be and B and that their frequency evolved in the most "optimistic" way (Fig. 6a), one obtains the evolution of $\mathrm{BeB}$ in the framework of a very simple chemical evolution model (Fig. 6b): the linearity is not really reproduced, the deviation between theory and observations becoming more important at low metallicities.

\section{Conclusions}

The basic points of this short overview may be summarised as follows:

i) Observational data in the solar neighborhood allow in principle to derive the past SFR history of that region. In practice (because of large dispersion in the age-metallicity relationship) they allow only to exclude high early SFRs, favouring a very slow building of the local disk. If this is achieved through infall of primordial composition, only small depletion factors of $\mathrm{D}(\sim 2-3)$ are obtained, favouring low primordial values of $\mathrm{D}$.

ii) Observations of the Milky Way disk suggest a high SFR efficiency in its inner regions. Simple toy-models that reproduce reasonably well those observations, predict an important $\mathrm{D}$ gradient in the disk; when measured 
by forthcoming experiments, the $\mathrm{D}$ profile will provide invaluable information on the past history of the disk.

iii) The SFR evolution of the Milky Way is presumably smoother than the observed "cosmic" one. However, the range of metal abundances observed in Ly $\alpha$ absorbers corresponds well to the range spanned by the disk of our Galaxy during its evolution; those systems may well be (proto) galactic disks. Finally, according to these simple models, D can be considerably depleted in inner disk zones, but only at low redshifts.

iv) The contribution of WR stars to the CR composition alleviates but does not solve the "enigma" of the linearity of Be and B vs. Fe. This enigma is confirmed by recent observations (Primas, this volume) and its solution will eventually offer new clues as to the origin and acceleration of CR.

\section{References}

1. Bloemen H., et al., 1994, A\&A Let., 281, L5

2. Casse M., Lehoucq R. \& Vangioni-Flam E., 1995, Nature, 373, 318

3. Duncan D., Lemke D. \& Lambert D., 1992, ApJ, 401, 584

4. Duncan D., et al., 1997, ApJ, in press

5. Edvardsson B., Andersen J., Gustafsson B., Lambert D., Nissen P., \& Tomkin J., 1993, A\&A, 275, 101

6. Fich M., \& Silkey M., 1991, ApJ, 366, 107

7. Galli D., Stanghellini L., Tosi M. \& Palla F., 1997, ApJ, in press

8. Geiss J., 1997, in "Primordial Nuclei and their Galactic Evolution", Eds. N. Prantzos, M. Tosi \& R. von Steiger (Kluwer), in press

9. Kennicut R., 1989, ApJ, 344, 685

10. Kroupa P., Tout C., \& Gilmore G., 1993, MNRAS, 262, 545

11. Madau P., 1997, in 7th International Origins Conference, in press

12. Maeder A. \& Meynet G., 1994, A\&A, 287, 803

13. Menneguzzi M., Audouze J., \& Reeves H., 1971, A\&A, 15, 337

14. Meyer JP, Elisson D. \& Drury J., 1997, ApJ, in press

15. Lu L., Sargent W. \& Barlow T., 1996, ApJ Suppl., 107, 475

16. Pagel B., 1997 Nucleosynthesis and Chemical Evolution, CUP

17. Pei Y. \& Fall M., 1995, ApJ, 454, 69

18. Pettini M., Smith L., King D. \& Husntead R., 1997, ApJ, 486, 665

19. Prantzos N., Casse M., \& Vangioni-Flam E., 1993, ApJ, 403, 630

20. Prantzos N. \& Aubert O., 1995, A\&A, 302, 69

21. Prantzos N., 1996, A\&A, 310, 106

22. Prantzos N. \& Silk J., 1997, in preparation

23. Rana N., 1991, ARAA, 29, 129

24. Reeves H., 1994, Rev. Mod. Phys., 66, 193

25. Rocha-Pinto H. \& Maciel W., 1996, MNRAS, 279, 447

26. Ryan S., et al., 1992, ApJ, 388, 184

27. Shaver P., McGee R., Newton L., Danks A. \& Pottasch S., 1983, MNRAS, 204, 53

28. Smartt S. \& Rolleston W., 1997 ApJ Let., 481, 47

29. Storri-Lombardi L., McMahon R. \& Irwin M., 1996, MNRAS, 283, 79

30. Tinsley B., 1980, Fund. Cosm. Phys., 5, 287

31. Tosi M., Steigman G., Matteucci F. \& Chiapinni C., 1997, ApJ, submitted

32. Woosley S. et al. 1990, ApJ, 356, 272

33. Wyse R. \& Silk J., 1989, ApJ, 379, 700

34. Wyse R. \& Gilmore G., 1995, AJ, 110, 2771 